\title{
Hybrid topological evolution of multi-singularity vortex beams: Generalized nature for helical-Ince-Gaussian and Hermite-Laguerre-Gaussian modes
}

\author{
Yijie Shen ${ }^{*}$ Yuan Meng, Xing Fu, and Mali Gong \\ State Key Laboratory of Precision Measurement Technology and Instruments, Center for Photonic and Electronic, \\ Department of Precision Instruments, Tsinghua University, Beijing, 100084, China
}

(Dated: November 7, 2018)

\begin{abstract}
A generalized family of scalar structured Gaussian modes including helical-Ince-Gaussian (HIG) and Hermite-Laguerre-Gaussian (HLG) beams is presented with physical insight upon a hybrid topological evolution nature of multi-singularity vortex beams carrying orbital angular momentum (OAM). Considering the physical origins of intrinsic coordinates aberration and the Gouy phase shift, a closed-form expression is derived to characterize the general modes in astigmatic optical systems. Moreover, a graphical representation, Singularities Hybrid Evolution Nature (SHEN) sphere, is proposed to visualize the topological evolution of the multi-singularity beams, accommodating HLG, HIG and other typical subfamilies as characteristic curves on the sphere surface. The salient properties of SHEN sphere for describing the precise singularities splitting phenomena, exotic structured light fields, and Gouy phase shift are illustrated with adequate experimental verifications.
\end{abstract}

\section{INTRODUCTION}

As distinctive structured light fields with phase singularities, optical vortices carrying orbital angular momentum (OAM) have hatched plenty of modern scientific applications in optical tweezers [1-3], optical communications [4, 5], quantum entanglement [6] 8] and nonlinear optics 9 11. Besides the classical Laguerre-Gaussian (LG) beams carrying integer OAM with a single phase singularity, the multi-singularity vortex beams carrying fractional OAM were also reported [12 17. The unique characteristics of multi-singularity beams and fractional OAM can be utilized to significantly increase capacity in optical communication systems [5, 18, 19, scale multiparticle manipulation technologies in optical tweezers [2, 3, 20, 21], drive advanced micro-opto-mechanics 22, flexibly shape light beam in 3-dimensional 23 25, and explore novel optical phenomena such as optical vortex knots [26 28] and spin-to-orbital conversion [29]. Several theoretical models serving as the eigensolutions of paraxial wave equation (PWE) were established to characterize multi-singularity beams, such as helical-Ince-Gaussian (HIG) modes [30, 31], elliptic LG beams [32, vortex HG beams 33 and generalized Gaussian beams [34, 35 . These models can be attributed to two families: HIG and Hermite-Laguerre-Gaussian (HLG) families, other modes except HIG modes constitute certain subfamilies of HLG family 36 38. HIG and HLG models are capable of describing multi-singularity beams in specific scopes of application. It was theoretically and experimentally verified that the multi-singularity HLG modes are transient states in HG-to-LG mode transforming process via astigmatic mode converter (AMC) [13, 15, 39 45]. Also, a large amount of multi-singularity vortex beams that cannot be well interpreted by HLG modes were studied based on HIG modes in terms of Ince-Gaussian (IG)

\footnotetext{
* shenyj15@mails.tsinghua.edu.cn
}

modes 46 49. As a family of scalar structured Gaussian modes (SSGMs) that are separable in elliptic coordinates, IG modes were also widely used to characterize special laser beams [30]. Meanwhile, researches showed that IG family has great advantage to characterize the general output modes in laser resonators, which accommodates HG and LG modes [31, 46, 50. Although both HLG and HIG models have their own peculiarities revealing different physical origins for describing multi-singularity modes, the variegated structured Gaussian beams can always show more complexities beyond the scope that classical theoretical modes can describe. Hence, it is significant to investigate generalized theories to illustrate the multi-singularity mode evolution and overcome the limitations of HIG and HLG models.

In this work, a generalized family of SSGMs including HLG and HIG modes is established to characterize the topological evolution of multi-singularity vortex beams sharing common physical origins in astigmatic optical systems. The description is based on a two-parameter mode family, one parameter represents Gouy phase difference introduced by astigmatism, and the other represents the degree of aberration of the elliptic intrinsic coordinates. Moreover, a novel graphical representation, Singularities Hybrid Evolution Nature (SHEN) sphere, is presented for vividly mapping the evolution of various multi-singularity modes. To verify our model, an abundance of examples are also demonstrated applying SHEN sphere for tailoring various intriguing multi-singularity modes.

\section{REVIEWS ON HLG AND HIG MODES}

\section{A. Multi-Singularity HLG modes}

A $\pi / 2$ AMC comprised of two cylindrical lenses leads to a Gouy phase shift difference of $\pi / 2$ at two orthogonal directions of input beam, which can transfer a $\mathrm{HG}_{n, m}$ beam with a $45^{\circ}$ inclined principal axes into a $\mathrm{LG}_{p, \ell}$ 

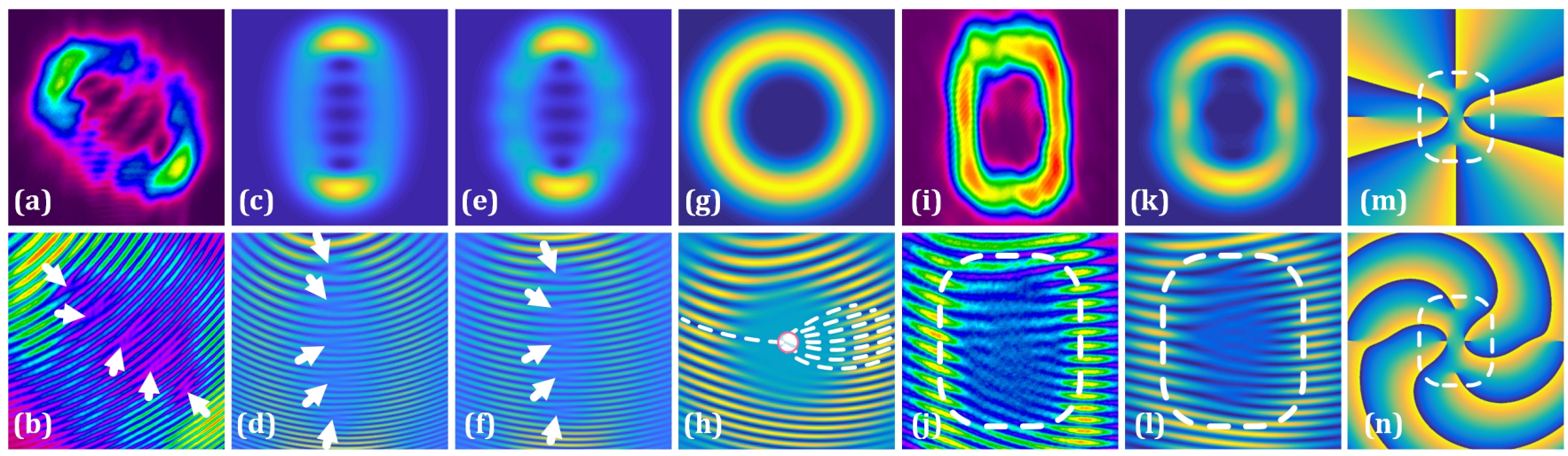

FIG. 1. The experimental and theoretical transverse patterns and interferograms: (a,b) the experimental output mode through $\pi / 2 \mathrm{AMC}$ when $\ell=5$ and $\alpha=30^{\circ}$ and $(\mathrm{c}, \mathrm{d})$ the corresponding theoretical interpretations by $\mathrm{HLG}_{0, \ell}\left(\alpha=30^{\circ}\right)$ mode; $(\mathrm{e}, \mathrm{f})$ anothor theoretical interpretations by $\mathrm{HIG}_{\ell, \ell}^{+}(\epsilon=4)$ for the linear-multi-singularity mode; $(\mathrm{g}, \mathrm{h})$ the theoretical LG ${ }_{0, \ell}$ mode when $\ell=6$ and $\alpha=45^{\circ} ;(\mathrm{i}, \mathrm{j})$ the experimental output mode when $\ell=6$ and $\alpha=45^{\circ} ;(\mathrm{k}, \mathrm{l})$ the theoretical HIG ${ }_{n, m}(\epsilon=9)$ mode, and phase distributions $(\mathrm{m})$ at beam waist and $(\mathrm{n})$ at a propagating distance.

beam, where $p=\min (n, m)$ and $\ell=n-m$, carrying OAM of $\ell \hbar$ per photon, $\ell$ is called topological charge. Basic mode matching conditions are required: (a) the cylindrical lenses with focal length of $f_{c}$ are separated by a distance of $d_{c}=f_{c} / \sqrt{2}$; (b) the input beam has a proper Rayleigh range of $z_{R}=(1+1 / \sqrt{2}) f_{c}$; (c) the beam waist locates at the center of two cylindrical lenses [39, 40. The mode conversion feature is revealed by Beijersbergen's equalities [40]:

$$
\begin{array}{r}
\mathrm{HG}_{n, m}\left(\frac{x+y}{\sqrt{2}}, \frac{x-y}{\sqrt{2}}, z\right) \\
=\sum_{K=0}^{N} b(n, m, K) \cdot \mathrm{HG}_{N-K, K}(x, y, z), \\
\mathrm{LG}_{p, \pm \ell}(x, y, z) \\
=\sum_{K=0}^{N}( \pm \mathrm{i})^{K} b(n, m, K) \cdot \mathrm{HG}_{N-K, K}(x, y, z),
\end{array}
$$

where $N=n+m$. Eq.(1) elucidates that a $45^{\circ}$ diagonal inclined $\mathrm{HG}_{n, m}$ mode can be spaned by the $\mathrm{HG}_{N-K, K}$ modes that constitute $N$-degenerate family with weight coefficients

$$
b(n, m, K)=\left.\sqrt{\frac{(N-K) !}{2^{N} n ! m ! K !}} \cdot \frac{\mathrm{d}^{K}}{\mathrm{~d} t^{K}}\left[(1-t)^{n}(1+t)^{m}\right]\right|_{t=0},
$$

then Eq. 22 reveals that a diagonal $\mathrm{HG}_{n, m}$ mode can be converted to $\mathrm{LG}_{p, \pm \ell}$ mode, where the phase shift term $( \pm \mathrm{i})^{K}$ is induced by $\pi / 2$ AMC. Assuming an arbitrary inclined angle $\alpha$ of the input $\mathrm{HG}_{n, m}$, the output mode can be expressed by HLG mode having linear splitting singularities (vortices array) with number of $|\ell|$, which is interpolated between HG and LG modes (for $|\ell|>1$ )
[36 38]:

$$
\begin{aligned}
\mathrm{HLG}_{n, m}(x, y, z \mid \alpha) & \\
= & \frac{1}{\sqrt{2^{N-1} n ! m !}} \exp \left(-\frac{r^{2}}{w^{2}}\right) \mathrm{HL}_{n, m}\left(\frac{\mathbf{r}}{\sqrt{\pi} w} \mid \alpha\right) \\
& \quad \exp \left[\mathrm{i} k z+\mathrm{i} k \frac{r^{2}}{2 R}-\mathrm{i}(m+n+1) \psi\right]
\end{aligned}
$$

where the Hermite-Laguerre (HL) polynomials

$$
\operatorname{HL}(\mathbf{r} \mid \alpha)_{n, m}=\left.\frac{\partial^{m}}{\partial s_{x}^{m}} \frac{\partial^{n}}{\partial s_{y}^{n}} \mathcal{G}(\mathbf{r}, \mathbf{s} \mid \alpha)\right|_{\mathbf{s}=\mathbf{0}},
$$

with generating function

$$
\mathcal{G}(\mathbf{r}, \mathbf{s} \mid \alpha)=\exp \left[-\left(\mathbf{U}^{*} \mathbf{s}\right)^{\mathrm{T}}(\mathbf{U} \mathbf{s})+2 \sqrt{2 \pi}\left(\mathbf{U}^{*} \mathbf{s}\right)^{\mathrm{T}} \mathbf{r}\right],
$$

where $\mathbf{r}=(x, y)^{\mathrm{T}}=(r \cos \varphi, r \sin \varphi)^{\mathrm{T}}, \mathbf{s}=\left(s_{x}, s_{x}\right)^{\mathrm{T}}$, $\mathbf{U}=\left(\begin{array}{cc}\cos \alpha & \mathrm{i} \sin \alpha \\ \mathrm{i} \sin \alpha & \cos \alpha\end{array}\right), k=2 \pi / \lambda, R(z)=\left(z_{R}^{2}+z^{2}\right) / z$, the $1 /$ e beam spot radius $w(z)=\left[2\left(z_{R}^{2}+z^{2}\right) /\left(z_{R} k\right)\right]^{1 / 2}$, Gouy phase $\psi(z)=\arctan \left(z / z_{R}\right)$, and $z_{R}$ is the Rayleigh length. When $\alpha=0$ or $\pi / 2$, the $\mathrm{HLG}_{n, m}$ mode is reduced to $\mathrm{HG}_{n, m}$ or $\mathrm{HG}_{m, n}$ mode; when $\alpha=\pi / 4$ or $3 \pi / 4$, the $\mathrm{HLG}_{n, m}$ mode is reduced to $\mathrm{LG}_{p, \pm \ell}$ mode. Fig.1 $1(a, b)$ shows the measured output mode and their interferogram patterns via a $\pi / 2$ AMC when the input was $\mathrm{HG}_{0, \ell}(\ell=5)$ mode inclined by $30^{\circ}$ in our experiment (see details in section 5.1), where a linear array of five singularities is illustrated, which are well interpreted by $\mathrm{HLG}_{0, \ell}\left(\alpha=30^{\circ}\right)$, as shown in Fig]1(c,d).

Considering that the mode matching conditions (a-c) are not strictly satisfied, i.e. the Gouy phase difference along two orthogonal directions is an arbitrary value $\beta \in$ $(-\pi, \pi]$, while the input is still a $45^{\circ}$ inclined HG mode, the output mode can be ascribed into the $\beta$-drived HLG 
family that

$$
\begin{aligned}
& \operatorname{HLG}_{n, m}^{\star}(x, y, z \mid \beta) \\
& \quad=\sum_{K=0}^{N} \mathrm{e}^{\mathrm{i} \beta K} b(n, m, K) \cdot \mathrm{HG}_{N-k, k}(x, y, z) .
\end{aligned}
$$

In AMC, $\beta$ is related to the $d_{c}$ and $f_{c}$ by [40]:

$$
\begin{aligned}
\beta & = \pm\left(\Delta \psi_{x}-\Delta \psi_{y}\right) \\
& = \pm 2\left(\arctan \sqrt{\frac{1+d_{c} / f_{c}}{1-d_{c} / f_{c}}}-\arctan \sqrt{\frac{1-d_{c} / f_{c}}{1+d_{c} / f_{c}}}\right),
\end{aligned}
$$

where the " \pm " denotes the interchangeability of $x, y$. For $\beta= \pm \pi / 2$, i.e. the case of $\pi / 2$ AMC, Eq.(7) is reduced to Eq. (2); for $\beta=\pi$ and $\beta=0$, the transformations represent the reversion and invariability respectively. In fact, $\mathrm{HLG}^{\star}$ and HLG represent the equivalent family but driven by different parameters, sharing the same physical origin of Gouy phase difference 41,43].

\section{B. Multi-Singularity HIG modes}

The HLG modes cannot completely cover SSGMs. There are other eigensolutions which are separable in elliptic coordinates, e.g. even and odd IG beams [30,

$$
\begin{aligned}
\mathrm{IG}_{u, v}^{e}(x, y, z \mid \epsilon)= & \frac{C_{\mathrm{IG}}}{w} C_{u}^{v}(\mathrm{i} \xi, \epsilon) C_{u}^{v}(\eta, \epsilon) \exp \left(-\frac{r^{2}}{w^{2}}\right) \\
& \exp \left[\mathrm{i} k z+\mathrm{i} k \frac{r^{2}}{2 R}-\mathrm{i}(u+1) \psi\right], \\
\mathrm{IG}_{u, v}^{o}(x, y, z \mid \epsilon)= & \frac{S_{\mathrm{IG}}}{w} S_{u}^{v}(\mathrm{i} \xi, \epsilon) S_{u}^{v}(\eta, \epsilon) \exp \left(-\frac{r^{2}}{w^{2}}\right) \\
& \exp \left[\mathrm{i} k z+\mathrm{i} k \frac{r^{2}}{2 R}-\mathrm{i}(u+1) \psi\right],
\end{aligned}
$$

which are also interpolated between HG and LG beams but separable in elliptic coordinates $(\xi, \eta)$ with ellipticity of $\epsilon$, where $C_{\mathrm{IG}}$ and $S_{\mathrm{IG}}$ are normalization constants, $C_{u}^{v}(\cdot, \epsilon)$ and $S_{u}^{v}(\cdot, \epsilon)$ are the even and odd Ince polynomials with indices of $(u, v)$. It can be noted that Gouy phase is a general term in a same degenerate state no matter for IG or HLG model, i.e. $\exp [-\mathrm{i}(u+1) \psi]=$ $\exp [-\mathrm{i}(m+n+1) \psi]=\exp [-\mathrm{i}(2 p+l+1) \psi] . \quad \xi \in$ $[0, \infty)$ and $\eta \in[0,2 \pi)$ are the radial and angular elliptic variables respectively, which are related to the Cartesian coordinates $(x, y)$ by

$$
(x, y)=[f(z) \cosh \xi \cos \eta, f(z) \sinh \xi \sin \eta],
$$

where $f(z)=w(z) \sqrt{\epsilon / 2}$. Eqs. 11. can be regarded as the parameter equation of ellipse or hyperbola family [schematics are illustrated in Fig.2(a,b)], which can cover the whole $\mathbb{R}^{2}$ plane driven by different ellipticity $\epsilon$.
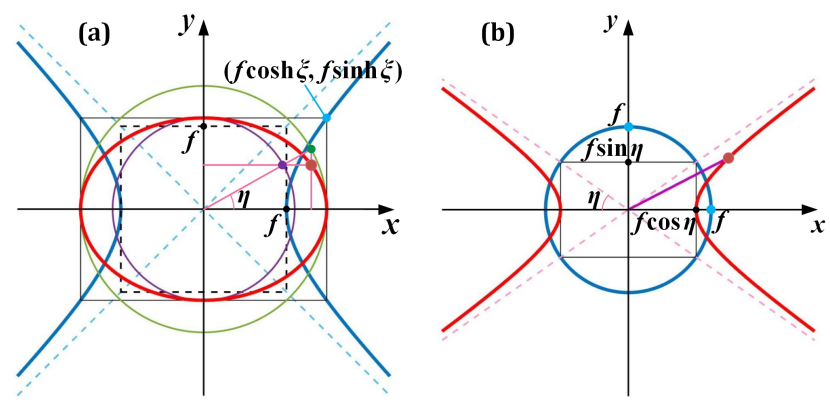

FIG. 2. The intrinsic coordinates system of IG beam can be regarded as a family of (a) ellipse or (b) hyperbola: (a) the ellipse with eccentric angle of $\eta$ and two semi-major axis lengths of $f \cosh \xi$ and $f \sinh \xi$ can cover the whole $\mathbb{R}^{2}$ plane driven by the point ( $f \cosh \xi, f \sinh \xi$ ) on a hyperbola; (b) the hyperbola with asymptotic line inclined by $\eta$ angle, semi-real axis lengths of $f \cos \eta$, and semi-imaginary axis lengths $f \sin \eta$ can cover the whole $\mathbb{R}^{2}$ plane driven by the point $(f \cos \eta, f \sin \eta)$ on a circle.

It has been proved that the eigen modes of simple spherical laser cavities are eigenfunctions of twodimensional quantum harmonic oscillators as the solutions of PWE [52 54], which can be analytically expressed as HG functions with Cartesian symmetry [55], LG functions with circular symmetry [56, IG functions with elliptic symmetry [57, and various distribution functions with other special intrinsic coordinates [58, 59]. The intrinsic elliptic coordinates tend to have circular symmetry when the ellipticity parameter $\epsilon \rightarrow 0$, and have Cartesian symmetry when $\epsilon \rightarrow \infty$ 31, 55,57. The recently proposed HIG beams, $\mathrm{HIG}_{u, v}^{ \pm}=\mathrm{IG}_{u, v}^{e} \pm \mathrm{i} \cdot \mathrm{IG}_{u, v}^{o}$, have described many multi-singularity beams breaking the limitation of HLG family [31, 46, 49. $\mathrm{HIG}^{ \pm}$model also has the potential to interprete a linear vortices array, such as the multi-singularity mode $\mathrm{HIG}_{\ell, \ell}^{ \pm}$with $\ell=5$, as shown in Fig 1 $1(e, f)$.

\section{Difficulties of HLG and HIG modes}

$\mathrm{HIG}^{ \pm}$and HLG modes can describe multi-singularity vortex beams or singularities splitting phenomena in AMC systems, however, both of which has limitations (see more evidences in section 5.2). Based on HLG model, doughnut-shape $\mathrm{LG}_{0, \ell}$ beam is expected to be obtained via $\pi / 2 \mathrm{AMC}$ for a input $45^{\circ}$ inclined $\mathrm{HG}_{0, \ell}$, as shown in Fig $1(\mathrm{~g}, \mathrm{~h})(\ell=6)$. However, we also observed a square-shape aberrant profile and nonlinear multiple singularities array, see Fig $1(i, j)$, which is beyond the scope of $\mathrm{HLG}$ and $\mathrm{HIG}^{ \pm}$model, even if the $\mathrm{AMC}$ was in vicinity of $\beta= \pm \pi / 2$ and $\alpha=\pi / 2 \pm \pi / 4$.

In next chapter, we will demonstrate that the elliptical aberration of the input HG modes plays a crucial role in the singularities splitting phenomenon. In other words, the topological evolution of singularities in AMC system 

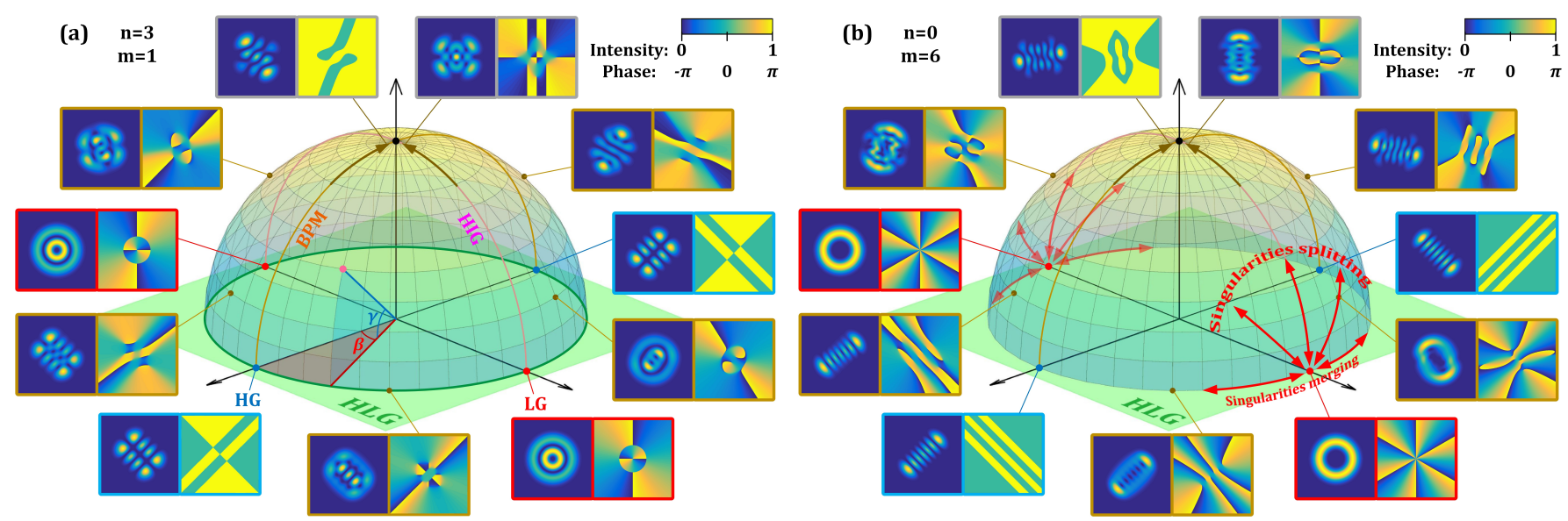

FIG. 3. SHEN spheres with orders of $(\mathrm{a})(n, m)=(3,1)$ and $(\mathrm{b})(n, m)=(0,6)$ with represented mode fields (phase) at selected points. The subfamily of HG, LG, HLG, HIG ${ }^{\star}$, BPM modes, and angles $\beta$ and $\gamma$ are marked in figure (a). The tendencies of singularities splitting and singularities merging are marked as double sided arrows in figure (b). See video illustrations of SHEN spheres for more cases: Visualization 1 for $(n, m)=(0,1)$, Visualization $2(0,2)$, Visualization $3(5,0)$, Visualization 4 $(6,0)$, Visualization $5(1,1)$, Visualization $\mathbf{6}(6,6)$, Visualization $\mathbf{7}(1,3)$, and Visualization $8(6,3)$.

is not only dominated by HLG description but also related to an IG aberration principle, coming down to a hybrid topological evolution nature. Moreover, we really observed the corresponding IG aberration evidences in our HG mode oscillator, which is illustrated in section 5.3 .

\section{HYBRID TOPOLOGICAL EVOLUTION NATURE}

\section{A. Hybrid HIG and HLG mode evolution}

For interpreting the exotic singularities splitting phenomenon, we propose a new definition of general HIG mode, which is also defined as the superposition of IG modes but in a more generalized way:

$$
\begin{aligned}
& \mathrm{HIG}_{n, m}^{\star}(x, y, z \mid \epsilon) \\
& \quad=\sum_{K=0}^{N}( \pm \mathrm{i})^{K} b(n, m, K) \cdot \mathrm{IG}_{N, K}^{\star}(x, y, z \mid \epsilon),
\end{aligned}
$$

where

$$
\begin{aligned}
& \mathrm{IG}_{N, K}^{\star}(x, y, z \mid \epsilon) \\
& \quad=\left\{\begin{array}{c}
(-\mathrm{i})^{K} \mathrm{IG}_{N, N-K}^{e}(x, y, z \mid \epsilon), \text { for }(-1)^{K}=1 \\
(-\mathrm{i})^{K} \mathrm{IG}_{N, N-K+1}^{o}(x, y, z \mid \epsilon), \text { for }(-1)^{K} \neq 1
\end{array} .\right.
\end{aligned}
$$

In other words, we replace the $\mathrm{HG}_{N-K, K}$ modes by the corresponding IG modes that can be degraded into $\mathrm{HG}_{N-K, K}$ modes in Eq. 22. When $\epsilon \rightarrow \infty$, the $\mathrm{IG}_{N, K}^{\star}$ mode is degraded into $\mathrm{HG}_{N-K, K}$ mode, and $\mathrm{HIG}^{\star}$ is degraded into $\mathrm{LG}_{p, \ell}$. Rather than HLG $[\mathrm{Fig}, 1(\mathrm{~g}, \mathrm{~h})]$ and
$\mathrm{HIG}^{ \pm}\left[\mathrm{Fig}[1(\mathrm{k}, \mathrm{l})]\right.$ models, the generalized HIG ${ }^{\star}$ model can successfully elucidate the exotic multi-singularity mode shown in Fig $1(i, j)$, where the precise fringe tracks induced by multiple singularities are highly consistent between experiment and theory. Fig $1(m, n)$ depicts the simulated phase at beam waist and at a propagating distance respectively, where the morphology of phase vortex solitons is clearly illustrated. It is noted that HLG is included as a subfamily in HIG ${ }^{\star}$ family, therefore, HIG $\star$ description is more felicitous for the actual singularities evolution details in AMC experiment.

Further considering the hybrid properties of $\mathrm{HIG}^{\star}$ and HLG modes, a more generalized model is established to elucidate the singularities hybrid evolution in AMC, based on the facts: (a) one usually cannot precisely control an exact phase difference of $\pi / 2$ in AMC but an arbitrary $\beta \in(-\pi, \pi]$; (b) the ellipticity can be evaluated by a parameter $\gamma \in[0, \pi / 2]$ as $\epsilon=2 / \tan ^{2} \gamma$ and the intrinsic elliptic coordinates interpolate between Cartesian coordinates and circular coordinates in a harmonic way:

$$
(x, y)=(w \cot \gamma \cosh \xi \cos \eta, w \cot \gamma \sinh \xi \sin \eta)
$$

Thus, a more generalized family of SSGMs called as SHEN family can be established as:

$$
\begin{aligned}
& \operatorname{SHEN}_{n, m}(x, y, z \mid \beta, \gamma)= \\
& \sum_{K=0}^{N} \mathrm{e}^{\mathrm{i} \beta K} b(n, m, K) \cdot \mathrm{IG}_{N, K}^{\star}\left(x, y, z \mid \epsilon=\frac{2}{\tan ^{2} \gamma}\right),
\end{aligned}
$$

SHEN family is reduced to HIG ${ }^{\star}$ family when $\beta= \pm \pi / 2$, reduced to HLG when $\gamma=0$, to HG when $(\beta, \gamma)=(0,0)$ or $(\pi, 0)$, and to $L G$ when $(\beta, \gamma)=( \pm \pi / 2,0)$ respectively. 

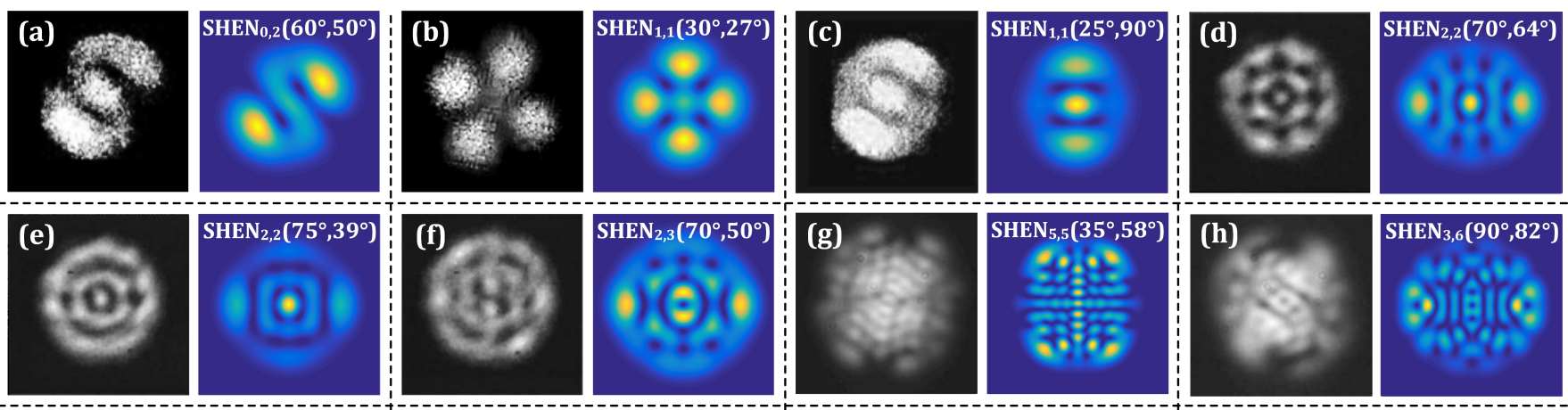

$\operatorname{SHEN}_{3,6}\left(90^{\circ}, \mathbf{8 2}^{\circ}\right)$
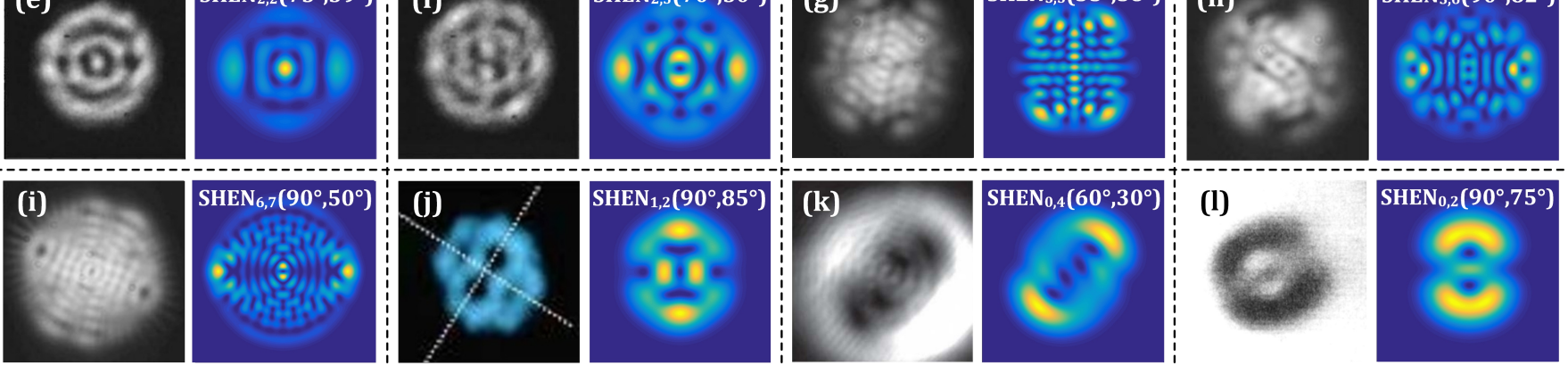

FIG. 4. The previously reported experimental modes (left) and the new interpretation by SHEN sphere (right): (a-c) the special modes in Ref.61], (d-f) Ref.62], (g-i) Ref.66], (j) Ref.64], (k) Ref. 65], (l) Ref. 66].

\section{B. SHEN sphere}

Hereinafter, we present a graphical representation to illustrate the topological evolution of the multi-singularity beams using SHEN sphere. $\operatorname{SHEN}_{n, m}(x, y, z \mid \beta, \gamma)$ mode is represented by the point with longitude of $\beta$ and latitude of $\gamma$ on the hemisphere surface. For instance, Figs 3(a,b) illustrates the SHEN spheres with indices of $(n, m)=(3,1)$ and $(n, m)=(0,6)$. Basic properties are noted as follow:

- The equator represents the family of $\mathrm{HLG}_{n, m}$ modes.

- The $\pm 90^{\circ}$ meridian represents the $\mathrm{HIG}_{n, m}^{*}$ family.

- The prime meridian represents the modes with binary phase distributions, while the binary phase modes (BPM) also have widespread applications such as light field modulation 60].

- Separated by the prime meridian, the eastern and western quarter-spheres represent two sets of modes which have inversion transformation (chirality) at antipodal points, for both phase and intensity distributions .

- The two points of intersection between the prime meridian and the equator represent the two inversed $\mathrm{HG}_{n, m}$ modes.

- The westernmost point and the easternmost point represent two $\mathrm{LG}_{p, \pm \ell}$ modes with opposite topological charges, which are the doughnut-shape vortex beams with a single singularity.

- The further away the mode is from the LG points, the more multi-splitting tendencies its singularities possess; while the nearer the mode is to the LG points, the more tendencies of merging into a single singularty they possess.

We note that there is a singular property on the north pole point of SHEN sphere: the represented mode has non-uniqueness but depends on the longitude as well (just like that you cannot tell a time on the north pole of earth). This property is in contrast to the Poincaré sphere 51, the polarization states described by which are not singular on the south and north poles. We stress that the functions of SHEN sphere and Poincaré sphere are different in that SHEN sphere characterizes the scalar fields structure and not polarization. With SHEN sphere, it is clear to explain the aforementioned nonlinear singularities array where an IG aberration of input beam leads to a SHEN mode away from the equator. More examples are shown in supplementary multimedia and can be found for detailed demonstration of the general evolution. Therefore, the singularities hybrid evolution nature of multi-singularity vortex beams can be clearly illustrated by SHEN sphere.

\section{Advantages of SHEN sphere}

SHEN sphere possesses great potential for interpreting multi-singularity SSGMs in more optical systems associated with general intrinsic symmetry and astigmatism effects besides the AMC systems. For instance, researchers have reported various exotic multi-singularity vortex beams in microchip lasers and astigmatic optical systems, but lack of theoretical descriptions [61 66]. The microchip lasers resonator can be seen as a generalized astigmatic optical system, where the self-reproductive modes are solved by PWE under certain intrinsic coordinates while the gain and thermal effects lead to astigmatism effects 61 64]. Fig 4 (a,b,c) shows the exotic S- 
shape, X-shape, and $\theta$-shape modes that were reported in a Nd:YAG Q-switched microchip laser 61. The authors provided the superposed expressions to describe them: (a) $\sqrt{0.4} \cdot \mathrm{e}^{\mathrm{i} \pi / 3} \cdot \mathrm{HG}_{0,2}+\sqrt{0.15} \cdot \mathrm{HG}_{2,0}+\sqrt{0.45}$. $\mathrm{HG}_{1,1}$, (b) $\sqrt{0.15} \cdot \mathrm{HG}_{0,2}+\sqrt{0.85} \cdot \mathrm{e}^{\mathrm{i} \pi / 3} \cdot \mathrm{HG}_{1,1}$, (c) $\sqrt{0.6} \cdot \mathrm{HG}_{0,2}+\sqrt{0.2} \cdot \mathrm{HG}_{2,0}+\sqrt{0.2} \cdot \mathrm{e}^{\mathrm{i} \pi / 3} \cdot \mathrm{HG}_{1,1}$. These modes can be interpreted in simpler ways under SHEN sphere model with (a) $\operatorname{SHEN}_{0,2}\left(\beta=60^{\circ}, \gamma=50^{\circ}\right)$, (b) $\operatorname{SHEN}_{1,1}\left(\beta=30^{\circ}, \gamma=27^{\circ}\right)$, (c) $\operatorname{SHEN}_{1,1}\left(\beta=25^{\circ}, \gamma=\right.$ $\left.90^{\circ}\right)$. Some complex multi-singularity optical vortices that can hardly be expressed by classical modes were previously reported in a microchip laser with a large Fresnel number 62, as shown in Fig 4(d,e,f), which can be explained by (d) $\operatorname{SHEN}_{2,2}\left(\beta=70^{\circ}, \gamma=64^{\circ}\right)$, (e) $\operatorname{SHEN}_{2,2}\left(\beta=75^{\circ}, \gamma=39^{\circ}\right)$, (f) $\operatorname{SHEN}_{2,2}\left(\beta=70^{\circ}, \gamma=\right.$ $\left.50^{\circ}\right)$. Also, more complex mode structures were obtained in a microchip self-Q-switched laser [63], as shown in Fig $4(\mathrm{~g}, \mathrm{~h}, \mathrm{i})$, which were described with a numerical model by authors, while the SHEN sphere can analytically interpret them by $(\mathrm{g}) \operatorname{SHEN}_{5,5}\left(\beta=35^{\circ}, \gamma=58^{\circ}\right)$, (h) $\operatorname{SHEN}_{3,6}\left(\beta=90^{\circ}, \gamma=82^{\circ}\right)$, (i) $\operatorname{SHEN}_{6,7}(\beta=$ $\left.90^{\circ}, \gamma=50^{\circ}\right)$. Moreover, the mode shown in Fig $4(\mathrm{j})$ reported in a single-frequency microchip lasers 64] can be interpreted by $\operatorname{SHEN}_{1,2}\left(\beta=90^{\circ}, \gamma=85^{\circ}\right)$; the modes shown in Fig $4(\mathrm{k}, 1)$ demonstrated in astigmatic optical systems [65, 66] can be interpreted by $\operatorname{SHEN}_{0,4}(\beta=$ $\left.60^{\circ}, \gamma=30^{\circ}\right)$ and $\operatorname{SHEN}_{0,2}\left(\beta=90^{\circ}, \gamma=75^{\circ}\right)$. Therefore, SHEN sphere provides a simple and powerful way to explore intriguing features of various structured vortex array beams in singular optics.

\section{DISCUSSION}

Taking advantages of the visualized illustrations and demonstration of clearer physical origins, SHEN sphere is expected to be widely utilized for tailoring structured light fields with multiple singularities matching the actual requirments in these vigorous scientific areas. Required arbitrary topological charges and distribution of multiple phase singularities can be found by scanning through the SHEN sphere of corresponding orders, and can be obtained by the realization of corresponding Gouy phase difference of $\beta$ and ellipticity of $\epsilon$. Therefore, SHEN sphere is more than a mathematical peculiarity, and is readily adapted as a powerful design tool for tailoring exotic structured light beams.

To sum up, a generalized family of SSGMs and a graphical representation are proposed for expressing the topological evolution of multi-singularity vortex beams. Comprehensive phenomena of multi-splitting singularities can be precisely characterized using our model. The SHEN modes are capable of interpreting multifarious SSGMs in general optical systems, naturally accounting for the classical families of HIG and HLG. The SHEN sphere opens a door for tailoring a large variety of structured beams with multiple singularities. Bearing in mind that several typical mode families such as HLG, HIG, BPM are

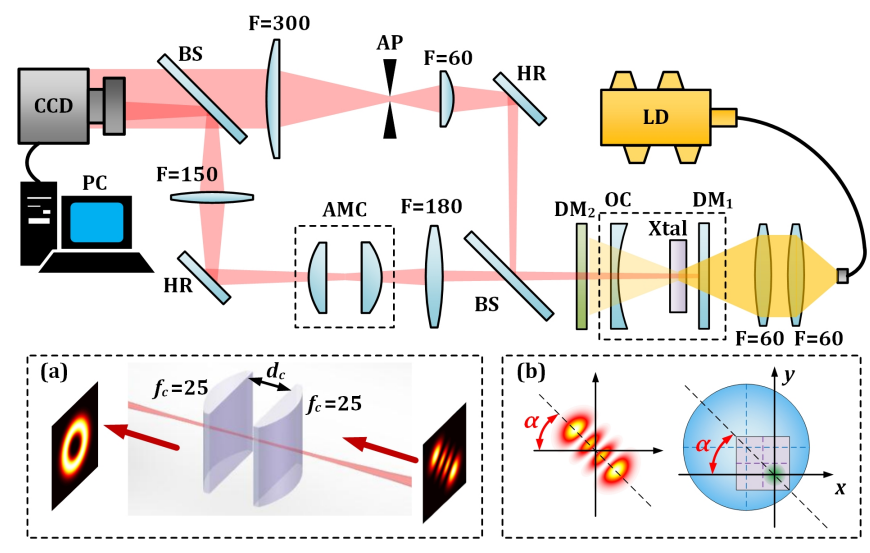

FIG. 5. Schematic of the experimental setup: LD, laser diode; Xtal: crystal; DM, dichroic mirror; OC, output coupler; BS, beam splitter; HR, high-reflective mirror; AP, aperture; AMC, astigmatic mode converter; CCD, charge coupled device; PC, personal computer. Inserts: (a) the details of the AMC with two cylindrical lenses; (b) the methods to control the inclined angle of the output HG mode; units: $\mathrm{mm}$.

respectively one-dimensional characteristic curves on the two-dimensional surface of SHEN sphere, the presented generalized theory significantly deepens the understanding of structured light fields.

\section{METHODS}

\section{A. Materials and instruments}

The experimental setup, as shown in Fig 5 includes two main parts: a diode-pumped solid-state laser oscillator for generating HG or IG mode; a Mach-Zehnder interferometer for generating orbital angular momentum (OAM) [via a astigmatic mode converter (AMC) in one arm, see Fig. 5(a)] and observing the phase singularities.

A $976 \mathrm{~nm}$ fiber-coupled laser diode (Hans TCS, core: $105 \mu \mathrm{m}$, NA: 0.22 , highest power: $110 \mathrm{~W})$ was used to pump a Yb:CALGO crystal (Altechna, $2 \times 2 \times 4 \mathrm{~mm}^{3}$, acut, 5 at.\%-doped) which was conductively cooling at $18^{\circ} \mathrm{C}$. The pump waist radius is about $200 \mu \mathrm{m}$ focused by a set of coupling lens including two identical convex lenses with focal length of $60 \mathrm{~mm}$. The laser was generated by a plano-concave resonator with a concave output coupling mirror (OC, radius of curvature of $300 \mathrm{~mm}$ ) and a flat dichroic mirror $\left[\mathrm{DM}_{1}\right.$, high-reflection $(\mathrm{HR})$ coated at $1040-1080 \mathrm{~nm}$ and antireflective (AR) coated at 976 $\mathrm{nm}]$. The cavity length is $16 \mathrm{~cm}$ and the distance between the crystal and $\mathrm{DM}_{1}$ is $2 \mathrm{~cm}$. We used the dualoff-axis method to generate high-order HG or IG modes: the mode order can be controlled by the off-axis distance and pump power, and the inclined angle of mode principal axes can be controlled by the off-axis direction [67], as illustrated in Fig. 1 (b). The $\mathrm{DM}_{2}$ (AR coated at 1040-1080 nm and HR coated at $976 \mathrm{~nm}$ ) was used to fil- 

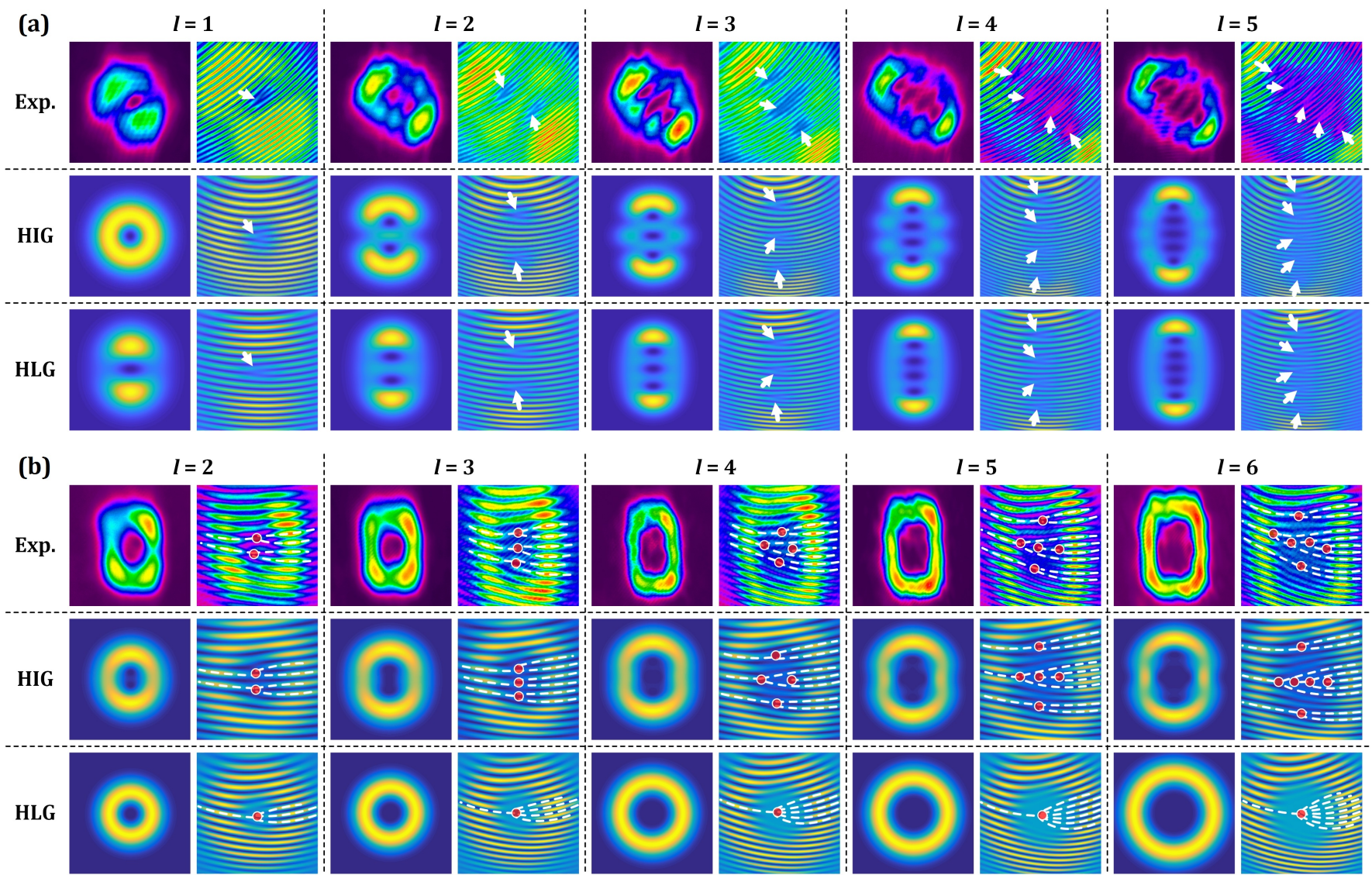

FIG. 6. (a) The first row: the experimental intensity pattern and interferograms for the output mode through AMC with various topological charge $\ell=1,2, \cdots, 5$ when inclined angle $\alpha=30^{\circ}$; the second row: the corresponding theoretical results of $\mathrm{HIG}_{\ell, \ell}^{+}(\epsilon=4)$; the third row: the corresponding theoretical results of $\mathrm{HLG}_{0, \ell}\left(\alpha=30^{\circ}\right)$. The positions of singularities are marked with by bold arrows in every interferograms. (b) The first row: the experimental results with various topological charge $\ell=2,3, \cdots, 6$ when inclined angle $\alpha=45^{\circ}$; the second row: the theoretical results of $\mathrm{HIG}_{0, \ell}^{\star}$; the third row: the theoretical results of $\mathrm{HLG}_{0, \ell}\left(\alpha=45^{\circ}\right)$. The positions of singularities are marked with by bold dots and the splitting tracks are marked by dash lines in every interferograms.

ter residual pump light. For the interferometer, the two arms were formed by two beam splitters $\left(\mathrm{BS}_{1}\right.$ and $\mathrm{BS}_{2}$, $45^{\circ}$ incidence, $\left.\mathrm{T}: \mathrm{R}=1: 9\right)$ and two $45^{\circ} \mathrm{HR}$ mirrors $\left(\mathrm{HR}_{1}\right.$ and $\mathrm{HR}_{2}$ ). In one arm, a telescope system was used to obtain near-plane wave as reference light, which includes two convex lenses with focal length of $60 \mathrm{~mm}$ and $300 \mathrm{~mm}$ respectively and an aperture. In another arm, the laser was focused into AMC by a convex lens with focal length of $180 \mathrm{~mm}$ for generating multi-singularity beam. The AMC was composed of two identical convex-plane cylindrical lenses with focal length of $f_{c}=25 \mathrm{~mm}$ separated by $d_{c}=35.4 \mathrm{~mm}\left(\sqrt{2} f_{c}\right)$. The multi-singularity beam was captured by a CCD camera (Spiricon, M2-200s) after being focused by a convex lens with focal length of $150 \mathrm{~mm}$ for observing the interference pattern.

\section{B. Generation of multi-singularity modes}

The multi-singularity beam is generated via the AMC and the input beam is the $\mathrm{HG}_{0, m}$ beam generated from the dual-off-axis resonator, which inevitably has IG aberration, as demonstrated in the next section. The corresponding perfect vortex beam converted by $\pi / 2$ converter should have the topological charge $\ell=m$. However, perfect $\pi / 2$ converter is difficult to obtain because there is usually a Rayleigh range mismatch. The first row of Fig 6 (a) shows the intensity profiles and interferograms for the case with inclined angle of $\alpha=30^{\circ}$ and $\ell$ from 1 to 5 . The HIG and HLG mode can describe these modes with linear splitting singularities array, i.e. $\mathrm{HIG}_{\ell, \ell}^{+}(\epsilon=4)$ and $\mathrm{HLG}_{0, \ell}\left(\alpha=30^{\circ}\right)$, the simulated results of which (intensity profiles and interferograms) are shown in the second and third row of Fig 6 (a) respectively. The HIG and HLG modes can describe the actual multi-singularity modes for $\ell=2,3,4,5$ with a good agreement (the linear distributed singularities are 




FIG. 7. The first row: the evolution of IG modes with various ellipticity gradually from infinity to zero, i.e. the $\mathrm{HG}$ mode is gradually transformed to the even or odd LG mode. The second row: the experimental results that an output HG mode can be transformed to IG mode with a certain ellipticity by slightly adjusting the laser resonator.

marked by bold arrows). However, for $\ell=1$, the HIG mode shows a doughnut shape that is deviated from the actual dual-hump profile, while HLG modes maintain a good agreement. For the case with the inclined angle of $\alpha=45^{\circ}$, as shown in the first row of Fig.6(b), a square-shape aberrant profile is obtained rather than a perfect LG vortex mode with a circular doughnut profile [as the third row of Fig $6(\mathrm{~b})]$, and the splitting singularities with number of $\ell$ can still be observed. The first reason is that the input $\mathrm{HG}$ mode has Ince-aberration, and the second one is the mode mismatch for realizing $\pi / 2$ converter. In these cases, the limitations of HLG modes are revealed: neither the square-shape aberration nor the splitting singularities phenomenon can be well described. For the sake of establishing more generalized model to describe the actual modes evolution in astigmatic optical systems, the aberration degree of intrinsic coordinates should be considered. Overcoming the limitations of $\mathrm{HLG}$ and $\mathrm{HIG}^{ \pm}$modes, the $\mathrm{HIG}^{\star}$ modes can give better interpretations for the singularities splitting and mode field aberration phenomenon, as shown in the second row of Fig, 6 (b).

\section{Eigen mode output in laser resonators}

The output mode in a laser resonator is usually a superior eigen mode in gain competition 68, the principle of which has guided many researchers to realize direct HG and LG output from resonator by using various selective pumping methods [67, 69 73. However, the intrinsic symmetry is actually influenced by complex factors such as cavity design, off-axis displacement [67, 72, 73, gain and thermal distributions [74, 75, as well as astigmatism effects of pump light [46, 50, 75], all of which can contribute to actual laser output mode. The IG modes, another form of eigenfunctions to the PWE that are separable under elliptic coordinates, can characterize more categories of output modes in laser resonator than $\mathrm{HG}$ and LG modes, and the HG and LG modes are just two extreme cases of IG modes 31, as illustrated in the first row of Fig. 7. The transition from an IG mode to a $\mathrm{HG}$ mode occurs when the elliptic coordinates tend to the Cartesian coordinates, i.e., when $\epsilon \rightarrow \infty$. The relation between $\mathrm{HG}$ and IG modes are given as below:

$$
\begin{aligned}
& \mathrm{HG}_{n, m}(x, y, z)= \\
& \left\{\begin{array}{c}
(-\mathrm{i})^{m} \mathrm{IG}_{m+n, n}^{e}(x, y, z \mid \epsilon \rightarrow \infty), \text { for }(-1)^{m}=1 \\
(-\mathrm{i})^{m} \mathrm{IG}_{m+n, n+1}^{o}(x, y, z \mid \epsilon \rightarrow \infty), \text { for }(-1)^{m} \neq 1
\end{array}\right.
\end{aligned}
$$

and the transition from an $\mathrm{IG}_{u, v}^{e, o}$ mode to a $\mathrm{IG}_{p, \ell}^{e, o}$ mode takes place when the elliptic coordinates approach circular cylindrical coordinates, i.e., when $\epsilon \rightarrow 0$. The relations between LG and IG modes are given by

$$
\mathrm{LG}_{p, \ell}^{e}(x, y, z)=(-\mathrm{i})^{m} \mathrm{IG}_{2 p+\ell, \ell}^{e}(x, y, z \mid \epsilon \rightarrow 0),
$$

$$
\mathrm{LG}_{p, \ell}^{o}(x, y, z)=(-\mathrm{i})^{m+1} \mathrm{IG}_{2 p+\ell, \ell}^{o}(x, y, z \mid \epsilon \rightarrow 0),
$$

where the even and odd LG modes, $\mathrm{LG}_{p, \ell}^{e}$ and $\mathrm{LG}_{p, \ell}^{o}$, are defined by replacing the Hilbert phase term $\exp (\mathrm{i} \ell \varphi)$ in normalized LG modes with $\cos (\ell \varphi)$ and $\sin (\ell \varphi)$ respectively [31]. The relation of the two kinds of $\mathrm{LG}$ modes are $\mathrm{LG}_{p, \ell}^{e}=\left(\mathrm{LG}_{p, \ell}+\mathrm{LG}_{p,-\ell}\right) / 2$ and $\mathrm{LG}_{p, \ell}^{o}=$ $-\mathrm{i}\left(\mathrm{LG}_{p, \ell}-\mathrm{LG}_{p,-\ell}\right) / 2$. The analytical expressions of $\mathrm{HG}_{n, m}$ and $\mathrm{LG}_{p, \ell}$ modes are given by:

$$
\begin{aligned}
\mathrm{HG}_{n, m}(x, y, z) & =\frac{C_{n, m}^{\mathrm{HG}}}{w} \exp \left(-\frac{x^{2}+y^{2}}{w^{2}}\right) H_{n}\left(\frac{\sqrt{2} x}{w}\right) H_{m}\left(\frac{\sqrt{2} y}{w}\right) \\
& \quad \exp \left[\mathrm{i} k z+\mathrm{i} k \frac{x^{2}+y^{2}}{2 R}-\mathrm{i}(m+n+1) \psi\right], \quad(19) \\
\mathrm{LG}_{p, \ell} & (r, \varphi, z) \\
= & \frac{C_{p, \ell}^{\mathrm{LG}}}{w}\left(\frac{\sqrt{2} r}{w}\right)^{|\ell|} \exp \left(-\frac{r^{2}}{w^{2}}\right) L_{p}^{|\ell|}\left(\frac{2 r^{2}}{w}\right) \exp (\mathrm{i} \ell \varphi) \\
& \exp \left[\mathrm{i} k z+\mathrm{i} k \frac{r^{2}}{2 R}-\mathrm{i}(2 p+|\ell|+1) \psi\right],
\end{aligned}
$$

where $(r, \varphi)=\left[\sqrt{x^{2}+y^{2}}, \arctan (y / x)\right], H_{n}(\cdot)$ is the Hermite polynomial of $n$-th order, $L_{p}^{\ell}(\cdot)$ is the generalized Laguerre polynomial with radial and azimuthal indices of $p$ and $\ell$, and the normalization constants for $\mathrm{HG}$ and LG modes are $C_{n, m}^{\mathrm{HG}}=\sqrt{2 /(\pi m ! n !)} \cdot 2^{-N / 2}$ and $C_{p, \ell}^{\mathrm{LG}}=\sqrt{2 p ! /[\pi(p+|\ell|) !]}=\sqrt{2 /(\pi m ! n !)} \cdot \min (n, m) !$.

\section{FUNDING}

The National Key Research and Development Program of China (Grant no. 2017YFB1104500); Natural Science Foundation of Beijing Municipality (4172030); Beijing Young Talents Support Project (2017000020124G044). 
[1] M. Padgett and R. Bowman, "Tweezers with a twist," Nat. Photonnics 5, 343-348 (2011).

[2] M. Woerdemann, C. Alpmann, and C. Denz, "Optical assembly of microparticles into highly ordered structures using Ince-Gaussian beams," Appl. Phys. Lett. 98, 111101 (2011).

[3] M. Woerdemann, C. Alpmann, M. Esseling, and C. Denz, "Advanced optical trapping by complex beam shaping," Laser Photonics Rev. 7, 839-854 (2013).

[4] A. E. Willner, H. Huang, Y. Yan, Y. Ren, N. Ahmed, G. Xie, C. Bao, L. Li, Y. Cao, Z. Zhao, J. Wang, M. P. J. Lavery, M. Tur, S. Ramachandran, A. F. Molisch, N. Ashrafi, and S. Ashrafi, "Optical communications using orbital angular momentum beams," Adv. Opt. Photonics 7, 66-106 (2015).

[5] J. Wang, "Advances in communications using optical vortices," Photonics Res. 4, B14-B28 (2016).

[6] R. Fickler, R. Lapkiewicz, W. N. Plick, M. Krenn, C. Schaeff, S. Ramelow, and A. Zeilinger, "Quantum Entanglement of High Angular Momenta," Science 338, 640643 (2012).

[7] R. Fickler, R. Lapkiewicz, M. Huber, M. P. J. Lavery, M. J. Padgett, and A. Zeilinger, "Interface between path and orbital angular momentum entanglement for highdimensional photonic quantum information," Nat. Commun. 5, 4502 (2014).

[8] O. S. Magaña-Loaiza, M. Mirhosseini, Robert M. Cross, S. M. H. Rafsanjani, and R. W. Boyd, "Hanbury Brown and Twiss interferometry with twisted light," Sci. Adv. 2, 1501143 (2016).

[9] R. N. Lanning, Z. Xiao, M. Zhang, I. Novikova, E. E. Mikhailov, and J. P. Dowling, "Gaussian-beampropagation theory for nonlinear optics involving an analytical treatment of orbital-angular-momentum transfer," Phys. Rev. A 96, 013830 (2017).

[10] F. Kong, C. Zhang, F. Bouchard, Z. Li, G. G. Brown, D. H. Ko, T.J. Hammond, L. Arissian, R. W. Boyd, E. Karimi, and P.B. Corkum, "Controlling the orbital angular momentum of high harmonic vortices," Nat. Commun. 8, 14970 (2017).

[11] D. Gauthier, P. R. Ribič, G. Adhikary, A. Camper, C. Chappuis, R. Cucini, L. F. DiMauro, G. Dovillaire, F. Frassetto, R. Géneaux, P. Miotti, L. Poletto, B. Ressel, C. Spezzani, M. Stupar, T. Ruchon, and G. De Ninno, "Tunable orbital angular momentum in high-harmonic generation," Nat. Commun. 8, 14971 (2017).

[12] J. B. Götte, K. O'Holleran, D. Preece, F. Flossmann, S. Franke-Arnold, S. M. Barnett, and M. J. Padgett, "Light beams with fractional orbital angular momentum and their vortex structure," Opt. Express 16, 9931006(2008).

[13] A. M. Nugrowati, W. G. Stam, and J. P. Woerdman, "Position measurement of non-integer OAM beams with structurally invariant propagation," Opt. Express 20, 27429-27441(2012).

[14] I. Martinez-Castellanos and J. C. Gutiérrez-Vega, "Vortex structure of elegant Laguerre-Gaussian beams of fractional order," J. Opt. Soc. Am. A 30, 2395-2400 (2013).

[15] A. M. Nugrowati and J. P. Woerdman, "Non-integer OAM beam shifts of Hermite-Laguerre-Gaussian beams," Opt. Commun. 308, 253-255(2013).
[16] J. C. Tung, H. C. Liang, T. H. Lu, K. F. Huang, and Y. F. Chen, "Exploring vortex structures in orbitalangularmomentum beams generated from planar geometric modes with a mode converter," Opt. Express 24, 22796-22805(2016).

[17] A. P. Porfirev, and S. N. Khonina, "Simple method for efficient reconfigurable optical vortex beam splitting," Opt. Express 24, 18722-18735(2017).

[18] J. A. Anguita, J. Herreros, and I. B. Djordjevic, "Coherent Multimode OAM Superpositions for Multidimensional Modulation," IEEE Photon. J. 6, 7900811 (2014).

[19] J. A. Anguita, J. Herreros, and I. B. Djordjevic, "Highdimensional modulation for coherent optical communications systems," Conference on Lasers and Electro-Optics, JTu2K.5 (2012).

[20] C. Kuo and S. Chu, "Numerical study of the properties of optical vortex array laser tweezers," Opt. Express 21, 26418-26431 (2013).

[21] J. E. Curtis, B. A. Koss, and D. G. Grier, "Dynamic holographic optical tweezers," Opt. Commun. 207, 169175 (2002).

[22] K. Ladavac and D. Grier, "Microoptomechanical pumps assembled and driven by holographic optical vortex arrays," Opt. Express 12, 1144-1149 (2004).

[23] X. Li, J. Chu, Q. Smithwick, and D. Chu, "Automultiscopic displays based on orbital angular momentum of light," J. Opt. 18, 085608 (2016).

[24] E. G. Abramochkin, and V. G. Volostnikov, "Spiral light beams," Phys. Uspekhi 47, 1177-1203 (2004).

[25] C. Chang, Y. Gao, J. Xia, S. Nie, and J. Ding, "Shaping of optical vector beams in three dimensions," Opt. Lett. 42, 3884-3887 (2017).

[26] M. R. Dennis, R. P. King, B. Jack, K. O'Holleran, and M. J. Padgett, "Isolated optical vortex knots," Nat. Phys. 6, 118-121 (2010).

[27] D. Kleckner and W. T. M. Irvine, "Creation and dynamics of knotted vortices," Nat. Phys. 9, 353-258(2013).

[28] S. J. Tempone-Wiltshire, S. P. Johnstone, and K. Helmerson, "Optical vortex knots - one photon at a time," Sci. Rep. 6, 24463(2016).

[29] R. C. Devlin, A. Ambrosio, N. A. Rubin, J. P. B. Mueller, F. Capasso, "Arbitrary spin-toorbital angular momentum conversion of light," Science 358, 896-901 (2017).

[30] M. A. Bandres and J. C. Gutiérrez-Vega, "Ince-Gaussian beams," Opt. Lett. 29, 144-146 (2004).

[31] M. A. Bandres and J. C. Gutiérrez-Vega, "Ince-Gaussian modes of the paraxial wave equation and stable resonators," J. Opt. Soc. Am. A 21, 873-880(2004).

[32] V. V. Kotlyar, S. N. Khonina, A. A. Almazov, and V. A. Soifer, "Elliptic Laguerre-Gaussian beams," J. Opt. Soc. Am. A 23, 43-56(2004).

[33] V. V. Kotlyar, A. A. Kovalev, and A. P. Porfirev, "Vortex Hermite-Gaussian laser beams," Opt. Lett. 40, 701-704 (2015).

[34] E. G. Abramochkin, and V. G. Volostnikov, "Generalized Gaussian beams," J. Opt. A: Pure Appl. Opt. 6, S157S161 (2004).

[35] Y. Wang, Y. Chen, Y. Zhang, H. Chen and S. Yu, "Generalised HermiteGaussian beams and mode transformations." J. Opt. 18, 055001 (2016). 
[36] T. Alieva, "Mode mapping in paraxial lossless optics," Opt. Lett. 30, 1461-1463 (2004).

[37] E. G. Abramochkin, and V. G. Volostnikov, Phys. "Generalized Hermite-Laguerre-Gauss Beams," Wave Phenom. 18, 14-22 (2010).

[38] E. Abramochkin, and T. alieva, "Closed-form expression for mutual intensity evolution of Hermite-LaguerreGaussian Schell-model beams," Opt. Lett. 42, 4032-4035 (2017).

[39] L. Allen, M. W. Beijersbergen, R. J. C. Spreeuw, and J. P. Woerdman, "Orbital angular momentum of light and the transformation of Laguerre-Gaussian laser modes," Phys. Rev. A 45, 8185-8189 (1992).

[40] M. W. Beijersbergen, L. Allen, H. E. L. O. van der Veen, and J. P. Woerdman, "Astigmatic laser mode converters and transfer of orbital angular momentum," Opt. Commun. 96, 123-132 (1993).

[41] L. Allen, "Matrix formulation for the propagation of light beams with orbital and spin angular momenta," Phy. Rev. E 60, 7497-7503 (1999).

[42] J. Courtial and M. J. Padgett, "Performance of a cylindrical lens mode converter for producing LaguerreGaussian laser modes," Opt. Commun. 159, 13-18(1999).

[43] A. T. O'Neil and J. Courtial, "Mode transformations in terms of the constituent Hermite-Gaussian or LaguerreGaussian modes and the variable-phase mode converter," Opt. Commun. 181, 35-45 (2000).

[44] M. J. Padgett and L. Allen, "Orbital angular momentum exchange in cylindrical-lens mode converters," J. Opt. B: Quantum Semiclass. Opt. 4, S17-S19 (2002).

[45] E. G. Abramochkin, E. V. Razueva, and V. G. Volostnikov, "Hermite-Laguerre-Gaussian beams in astigmatic optical systems," Proc. SPIE 7009, 70090M (2008).

[46] T. Ohtomo, S. Chu, and K. Otsuka, "Generation of vortex beams from lasers with controlled Hermite- and InceGaussian modes," Opt. Express 16, 5082-5094(2008).

[47] S. Chu, C. Yang, and K. Otsuka, "Vortex array laser beam generation from a Dove prism-embedded unbalanced Mach-Zehnder interferometer," Opt. Express 16, 19934-19949 (2008).

[48] J. B. Bentley and J. A. Davis, "Generation of helical Ince-Gaussian beams with a liquid-crystal display," Opt. Lett. 31, 649-651(2006).

[49] K. Otsuka and S. Chu, "Generation of vortex array beams from a thin-slice solid-state laser with shaped wide-aperture laser-diode pumping," Opt. Lett. 34, 1012 (2009).

[50] U. T. Schwarz, "Observation of Ince-Gaussian modes in stable resonators," Opt. Lett. 29, 1870-1872 (2004).

[51] G. Milione, H. I. Sztul, D. A. Nolan, and R. R. Alfano, "Higher-Order Poincaré Sphere, Stokes Parameters, and the Angular Momentum of Light," Phys. Rev. Lett. 107, 053601 (2011).

[52] D. Dragoman and M. Dragoman, Quantum-Classical Analogies (Springer, 2004).

[53] V. Bužek and T. Quang, "Generalized coherent state for bosonic realization of SU(2) Lie algebra," J. Opt. Soc. Am. B 6, 2447-2449 (1989).

[54] G. S. Agarwal and J. Banerji, "Entanglement by linear SU(2) transformations: generation and evolution of quantum vortex states," J. Phys. A 39, 11503-11519 (2006).

[55] M. A. Bandres and J. C. Gutiérrez-Vega, Opt. Lett. 32, "Cartesian beams," 3459-3461 (2007).
[56] M. A. Bandres and J. C. Gutiérrez-Vega, Opt. Lett. "Circular beams," 33, 177-179 (2008).

[57] M. A. Bandres and J. C. Gutiérrez-Vega, "Elliptical beams," Opt. Express 16, 21087-21092 (2008).

[58] J. C. Tung, Y. H. Hsieh, T. Omatsu, K. F. Huang, and Y. F. Chen, "Generating laser transverse modes analogous to quantum Green's functions of two-dimensional harmonic oscillators," Photonics Res. 5, 733-739 (2017).

[59] M. A. Alonso and M. R. Dennis, "Ray-optical Poincaré sphere for structured Gaussian beams," Optica 4, 476486 (2017).

[60] M. Hoffmann, I. N. Papadopoulos, and B. Judkewitz, "Kilohertz binary phase modulator for pulsed laser sources using a digital micromirror device," Opt. Lett. 43, 22-25 (2018).

[61] M. Wei, C. Chen, and K. Tu, "Spatial and temporal instabilities in a passively Q-switched Nd:YAG laser with a $\mathrm{Cr}^{4+}$ :YAG saturable absorber," Opt. Express 12, 39733980 (2004).

[62] Y. F. Chen and Y. P. Lan, "Transverse pattern formation of optical vortices in a microchip laser with a large Fresnel number," Phys. Rev. A 65, 013802 (2001).

[63] J. Dong and K. Ueda, "Observation of repetitively nanosecond pulse-width transverse patterns in microchip self-Q-switched laser," Phys. Rev. A 73, 053824 (2006).

[64] T. Ohtomo, K. Kamikariya, K. Otsuka, and S. Chu, "Single-frequency Ince-Gaussian mode operations of laser-diode-pumped microchip solid-state lasers," Opt. Express 15, 10705-10717 (2007).

[65] S. G. Reddy, S. Prabhakar, A. Aadhi, J. Banerji, and R. P. Singh, "Propagation of an arbitrary vortex pair through an astigmatic optical system and determination of its topological charge," J. Opt. Soc. Am. A 31, 12951302 (2014).

[66] A. B. Coates, C. O. Weiss, C. Green, E. J. D'Angelo, J. R. Tredicce, M. Brambilla, M. Cattaneo, L. A. Lugiato, R. Pirovano, and F. Prati, "Dynamical transverse laser patterns. II. Experiments," Phys. Rev. A 49, 1452-1466 (1994).

[67] Y. Shen, Y. Meng, X. Fu, and M. Gong, Opt. Lett. "Wavelength-tunable Hermite-Gaussian modes and an orbital-angular-momentum-tunable vortex beam in a dual-off-axis pumped Yb:CALGO laser" 43, 291-294 (2018).

[68] W. Koechner, Solid-State Laser Engineering, 6th ed. (Springer, New York, 2006).

[69] Y. F. Chen and Y. P. Lan, "Dynamics of the Laguerre Gaussian $\mathrm{TEM}_{0, l}^{*}$ mode in a solid-state laser," Phys. Rev. A 63, 063807 (2001).

[70] S. Ngcobo, K. Aït-Ameur, N. Passilly, A. Hasnaoui, and A. Forbes, "Exciting higher-order radial LaguerreGaussian modes in a diode-pumped solid-state laser resonator," Appl. Opt. 52, 2093-2101 (2013).

[71] A. Hu, J. Lei, P. Chen, Y. Wang, and S. Li, "Numerical investigation on the generation of high-order LaguerreGaussian beams in end-pumped solid-state lasers by introducing loss control," Appl. Opt. 53, 7845-7853 (2014).

[72] H. Laabs and B. Ozygus, "Excitation of Hermite Gaussian modes in end-pumped solidstate lasers via off-axis pumping," Opt. Laser Technol. 28, 213-214 (1996).

[73] Y. F. Chen, T. M. Huang, C. F. Kao, C. L. Wang, and S. C. Wang, "Generation of Hermite-Gaussian modes in fiber-coupled laser-diode end-pumped lasers," IEEE J. Quantum Electron. 33, 1025-1031 (1997). 
[74] X. Yan, Q. Liu, D. Wang, and M. Gong, "Combined guiding effect in the end-pumped laser resonator," Opt. Express 19, 6883-6902 (2011).
[75] I. Tamer, S. Keppler, M. Hornung, J. Körner, Joachim Hein, and M. C. Kaluza, "Spatio-Temporal Characterization of Pump-Induced Wavefront Aberrations in $\mathrm{Yb}^{3+}$. Doped Materials," Laser Photonics Rev. 1700211 (2017). 